\title{
Research Paper \\ Development of a Model to Predict Emotional Failure in Students According to Per- sonal Factors and Initial Maladaptive Schemas and Quality of Love Relationships
}

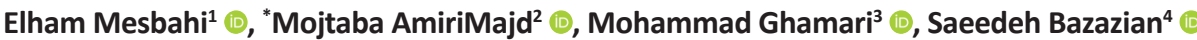

1. PhD. Candidate in Consulting, Department of Consulting, Faculty of Humanities, Abhar Branch, Islamic Azad University, Abhar, Iran

2. PhD. in Psychology and Exceptional Children Education, Associate Professor, Department of Psychology, Faculty of Humanities, Abhar Branch, Islamic Azad University, Abhar, Iran.

3. PhD. in Consulting, Assistant Professor, Department of Consulting, Faculty of Humanities, Abhar Branch, Islamic Azad University, Abhar, Iran.

4. PhD. in Health Psychology, Associate Professor, Department of Psychology, Faculty of Humanities, Abhar Branch, Islamic Azad University, Abhar, Iran.

\begin{tabular}{|c|c|}
\hline & ditation Mesbahi E, AmiriMajd M, Ghamari M, Bazazian S. Presenting a Model for Predicting Love Blight Syndrome in \\
\hline 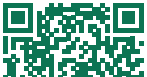 & $\begin{array}{l}\text { Students Based on Personality Traits, Initial Maladaptive Schemas and the Quality of Attachment Relationships. Iranian Journal } \\
\text { of Psychiatry and Clinical Psychology. 2019; 25(1):42-55. http://dx.doi.org/10.32598/ijpcp.25.1.42 }\end{array}$ \\
\hline rystis & doi'http://dx.doi.org/10.32598/ijpcp.25.1.42 \\
\hline
\end{tabular}

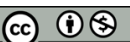

Received: 06 Aug 2018

Accepted: 17 Nov 2018

Available Online: 01 Apr 2019

Keywords:

Love Trauma, Quality

of attachment rela-

tionships, Personality

characteristics, Early

maladaptive schemes

\section{A B STRACT}

Objectives Love trauma is a common phenomenon among university students which causes educational and psychological problems in them. This study aimed to determine the role of personality traits, early maladaptive schemes, and the quality of attachment relationships in predicting Love Trauma (LT) syndrome.

Methods In this correlational study, the statistical population consisted of all patients referring to counseling centers of Iran University of Medical Sciences, Tehran University, and Islamic Azad University of Roudehen branch, with a complaint of emotional breakdown. Using purposeful sampling method, a total of 200 patients were selected as study samples. They were screened based on the cut-off point of Love Trauma Index (LTI). Data analysis was conducted using Structural Equation Modeling (SEM) method in LISREL Software.

Results SEM model showed a root mean square error of 0.047 which indicate good model fit.

Conclusion The provided model for predicting LT syndrome was a good fitting model. Personality traits, early maladaptive schemes, and the quality of attachment relationships could predict LT syndrome.

\section{Extended Abstract}

\section{Introduction}

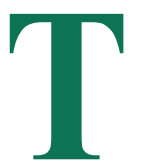

his research is conducted to determine the role of personal factors, initial maladaptive schemas, and quality of love relationships to predict the signs of emotional failure.

Our understanding of a happy and healthy adult is an individual who is capable of loving, in other words, establish- ing and maintaining long-term relationships [1]. However, after emotional failure, every individual experiences the symptoms of failure differently. The key dilemma is that a failure in a love relationship is associated directly with the decreased level of welfare [3], life satisfaction [4], anger and grief [5].

Researches show that personality traits [1] are among the factors influencing love relationships. Personality traits can be defined as dimensions of individual differences in the form of stable patterns of thinking, emotional, and behav-

a.

* Corresponding Author:

Mojtaba AmiriMajd, PhD.

Address: Department of Psychology, Faculty of Humanities, Abhar Branch, Islamic Azad University, Abhar, Iran.

Tel: +98 (912) 6983546

E-mail: amirimajd@yahoo.com 
ioral patterns [12]. One of the factors influencing personality characteristics is the style and quality of attachment [16]. Emotional attachment is a deep emotional bond between a person and a specific individual in his or her life. These studies on the role of personality traits, initial maladaptive schemas, and the quality of attachment relationships in predicting the emotional failure symptoms indicate that these factors are associated with the effects of emotional failure and can predict it [11]. It also suggests a relationship between personality traits and attachment styles with emotional failure [23].

\section{Method}

The present study has a correlational design. The statistical population consisted of all mental patients who were referred to counseling centers of Iran University of Medical Sciences, Tehran University, and Roudehen Azad University with a complaint of emotional failure. The sampling method was purposive and 200 samples with a complaint of love failure were recruited. The subjects responded to the questionnaires and were screened based on the cut-off scores for the emotional failure symptoms on the score scale. The obtained data were analyzed using structural equation modeling and LISREL software (Figure 1).

\section{Results}

The Mean \pm SD age of the participants was $15.23 \pm 4.52$ years. The findings indicate that the highest mean subscale value of early maladaptive schemas are related to

Table 1. The goodness of fit in the structural model

\begin{tabular}{ccccccc}
\hline Fitness Indicator & RMSEA & GFI & AGFI & NFI & NNFI & IFI \\
\hline Acceptable values & $<0.1$ & $>0.9$ & $>0.9$ & $>0.9$ & $>0.9$ & $0-1$ \\
Evaluated values & 0.047 & 0.95 & 0.93 & 0.96 & 0.98 & 0.91 \\
\hline & & & & $\begin{array}{l}\text { Iranian Journal of } \\
\text { PSYCHIATRY AND CUINICAL PSYCHOLOGY }\end{array}$
\end{tabular}

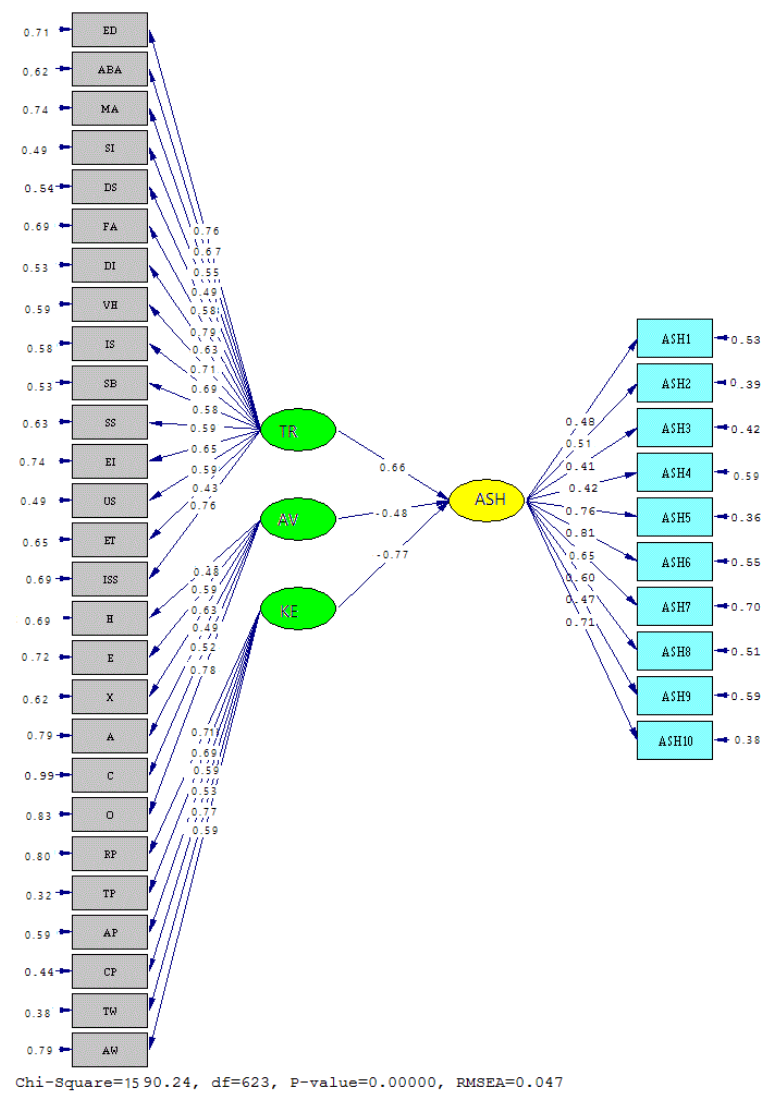

Figure 1. Results of structural equation modeling confirmation 
rigorous/defective and the lowest average relates to dependency/incompetence. Furthermore, the highest mean of the subscale of personality characteristics was related to excitability and the lowest mean to honesty/modesty. Also, the highest mean subscale of attachment relationships is associated with the relations with peers and the lowest to peer alienation.

Based on the findings, the early maladaptive schemas, personality traits, and the attachment quality have a significant relationship with emotional failure symptoms and the structural equation model shows a good fitness with a root mean square approximation index of 0.047 (Table 1). This means that the proposed model for the predictive elements of emotional failure symptoms fits with the inputs and personality characteristics, initial maladaptive schemas, and the quality of attachment relationships could predict the emotional failure symptoms. Furthermore, it shows that variables of extroversion, excitability, peer trust, sacrifice, abandonment/unsustainability, and modesty/honesty are the best determinants of emotional failure symptoms.

The results of this study indicate that the variable of emotional failure symptoms increases as the peer trust variable decreases. Moreover, self-sacrifice and abandonment/instability schemas predict the severity of emotional failure symptoms. Therefore, it seems that a reduction in the variable of self-sacrifice increases the severity of emotional failure symptoms while an increase in abandonment/instability variable increases the severity of emotional failure symptoms variable. The results of this study show that the variables for extroversion, excitement, and honesty/humility predict the emotional failure symptoms. With a decrease in the extroversion and honesty/humility variables and with an increase in the excitability variable, the emotional failure symptoms increases.

\section{Discussion}

With regard to a comprehensive outlook and in response to the key question of this research, we can conclude that our presented model fits properly so that regarding the relationship between predictive variables and the intensity of the emotional failure symptoms, it could predict the criterion variable. Maladaptive schemas such as self-sacrifice, abandonment/ instability, emotional deprivation, merit/ manners have the largest share in predicting the criterion variable. Among the characteristics of personality; extroversion, excitability and honesty/modesty; and among the relationship qualities; attachment, alienation of peers and trust in peers could properly predict the severity of emotional failure symptoms.

\section{Ethical Considerations}

\section{Compliance with ethical guidelines}

All ethical principles were considered in this article. The participants were informed about the purpose of the research and its implementation stages; they were also assured about the confidentiality of their information; Moreover, They were allowed to leave the study whenever they wish, and if desired, the results of the research would be available to them.

\section{Funding}

The present paper was extracted from the $\mathrm{PhD}$. dissertation of the first author, in Department of Psychology, Faculty of Humanities, Abhar Branch, Islamic Azad University.

\section{Authors contributions}

Conceptualization: All authors; Methodology: All authors; Validation: All authors; Investigation: All authors; Writing-original draft preparation: Elham Mesbahi; Writing-review \& editing: Elham Mesbahi, Mojtaba AmiriMajd; and Visualization, supervision, project administration: Mojtaba AmiriMajd.

\section{Conflict of interest}

The authors declared no conflict of interest. 


\title{
تدوين مدلى براى ييشبينى نشانعان ضربه عشقى در دانشجويان بر اساس ويثَّى هاي شخصيتى،

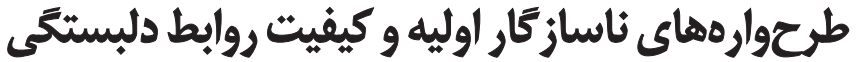

\author{
الهام مصباحى' هـ، "مجتبى اميرى مجد" هـ، محمد قمرى" هـ، سعيده بزازيان" \\ 1 - دانشجوى دكتراي مشاوره، كروه مشاوره، دانشكده علوم انسانى، واحد ابهر، دانشعاه آزاد اسلامى، ابهر، ايران.

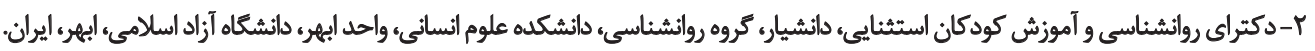

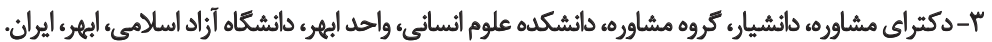

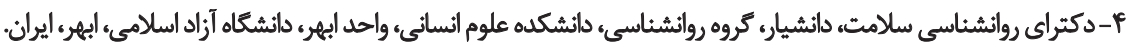

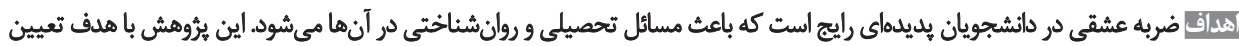

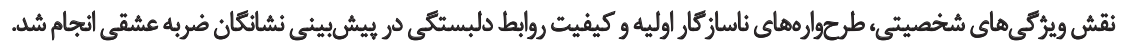

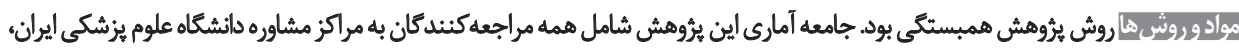

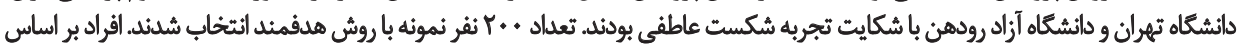

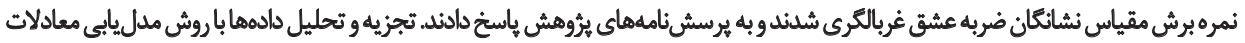

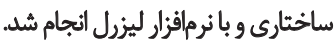

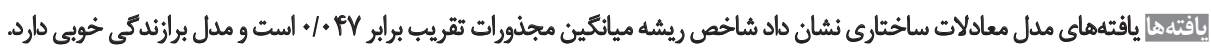

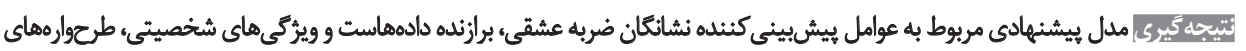

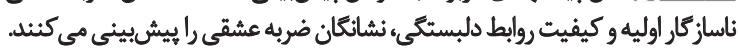

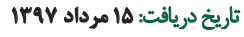

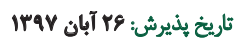
تاريخ انتشار: rا فرودين

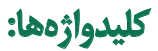

نشائَان ضربه عشقيى،

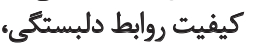

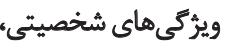
طرحوارههاى ناساز كار اوليه

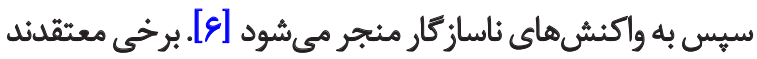

مقدمه

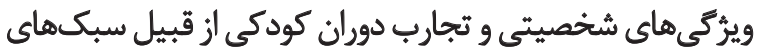

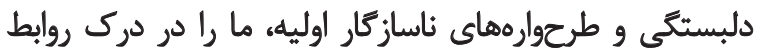

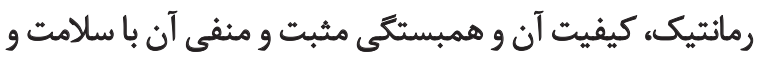

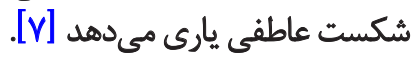

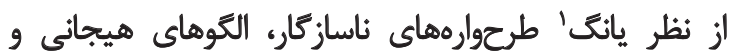

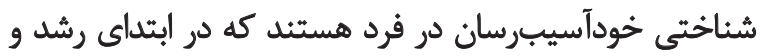

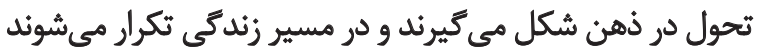

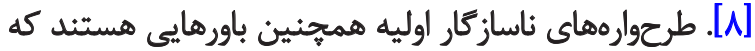

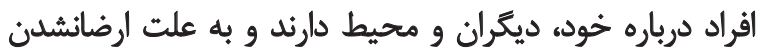

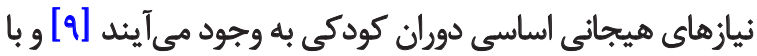

وضعيت سلامت روان رابطه دارند [. [1].

1. Young

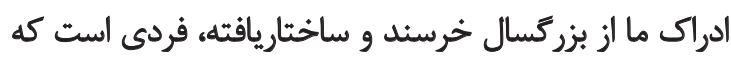

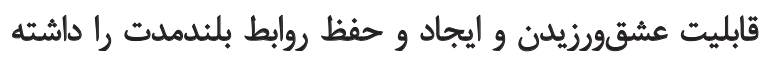

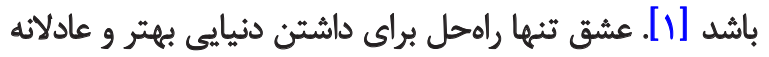

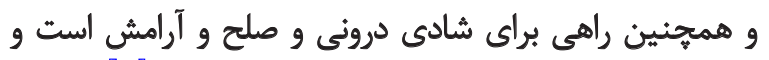

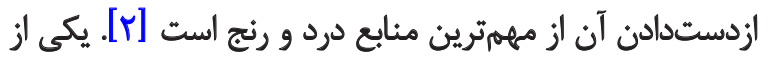

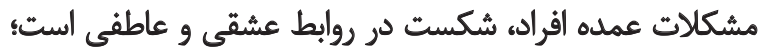

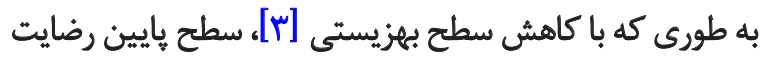

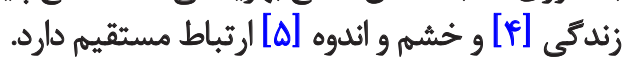

بعد از شكست عاطفى، افراد يك سرى از نشانههاى شكست

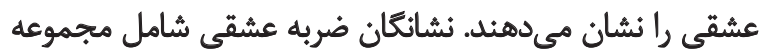

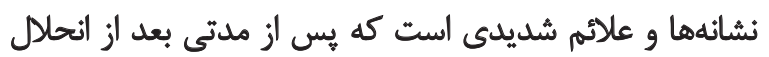

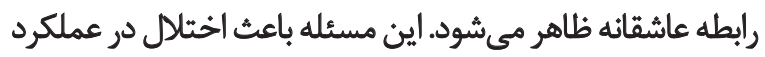

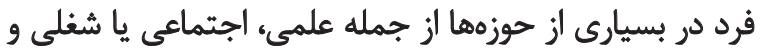

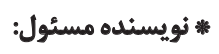

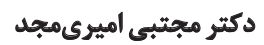

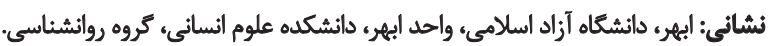
تلفن: يست الكترونيكي: amirimajd@yahoo.com 


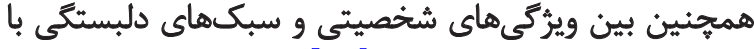

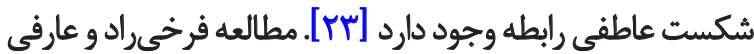

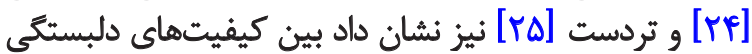

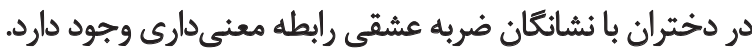

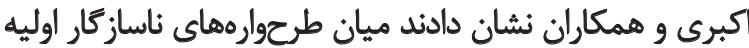

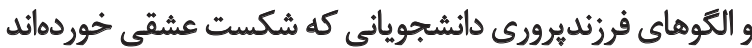

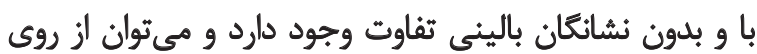

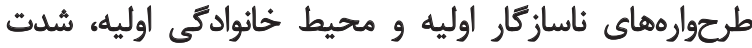

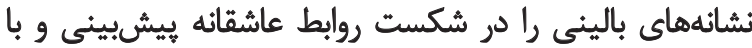

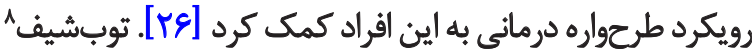

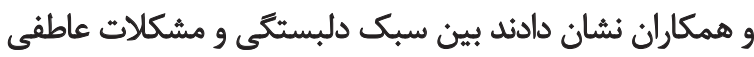

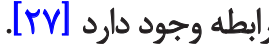

رولافس و ومكاران نشان دادند انواع مختلف طرحوارههاي

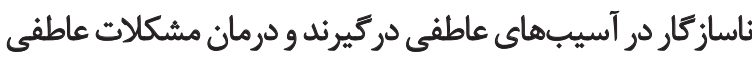

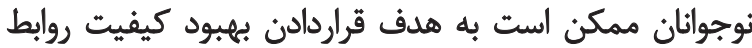

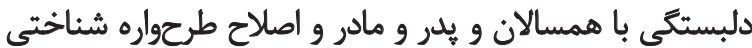

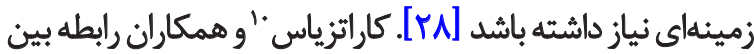

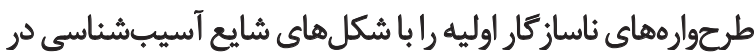

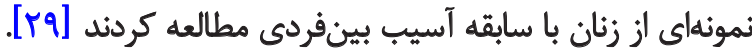

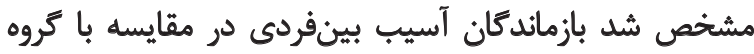

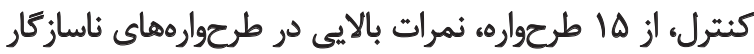

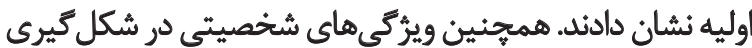

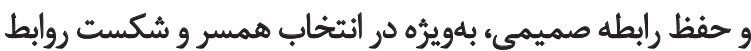

عاطفى مهم هستند [-•r].

با توجه به اهميت آشنايى و رابطه عاطفى با جنس مخالف

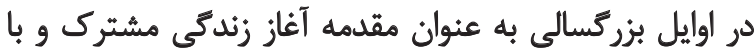

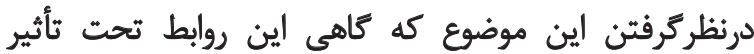

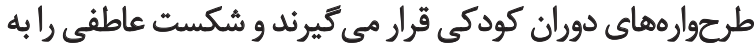

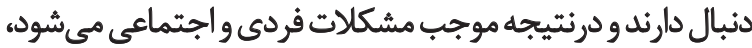

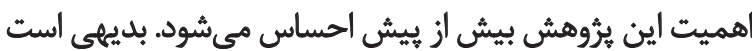

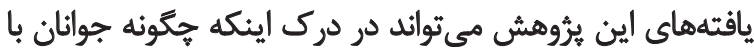

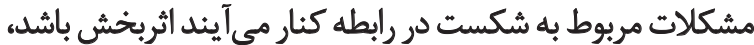

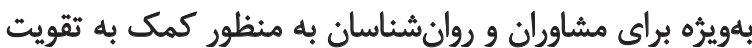

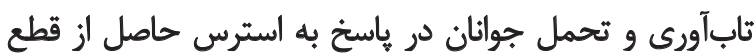

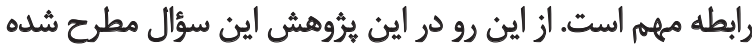

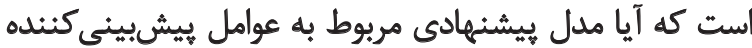

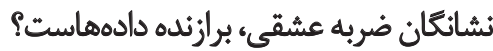

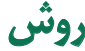

اين يثوهش از نوع همبستكى است و جامعه آمارى آن شامل

8. Taube-Schiff

9. Roelofs

10. Karatzias

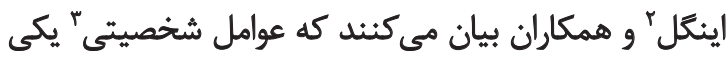

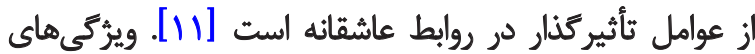

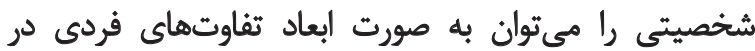

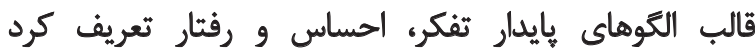

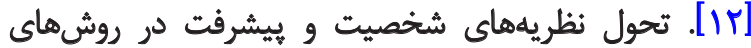

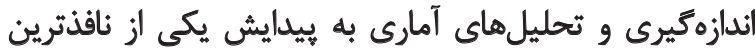

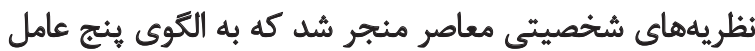

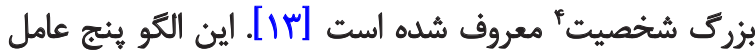

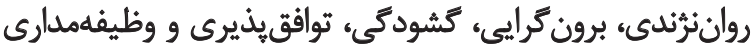
را شامل مىشود [1f]

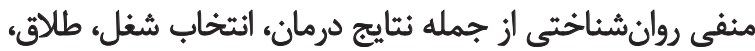

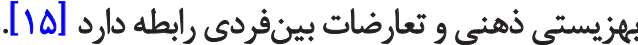

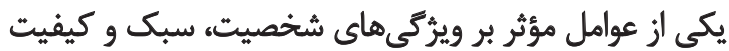

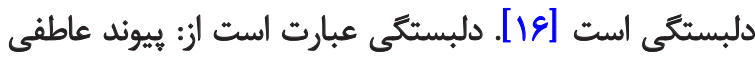

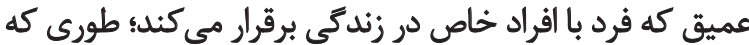

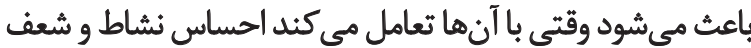

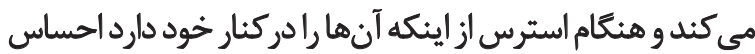

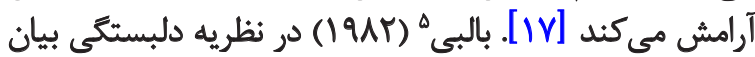

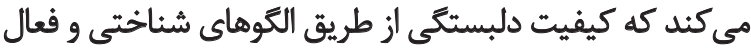

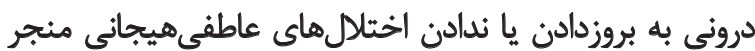

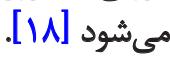

درواقع سبكهاي دلبستكى توان بيشبينى تنظيم هيجاني را خواهند داشت [19]

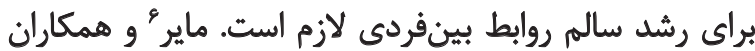

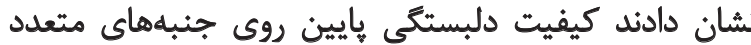

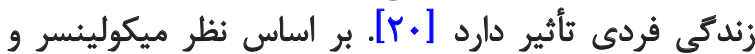

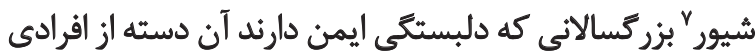

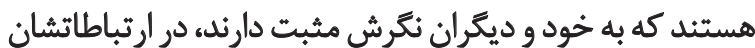

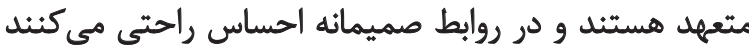

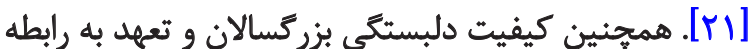

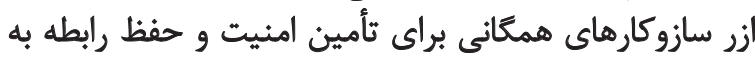

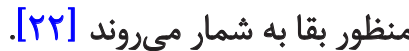

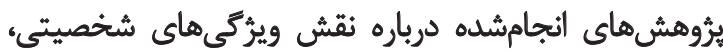

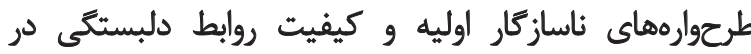

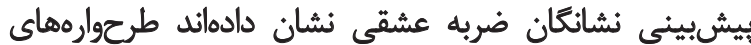

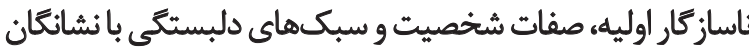

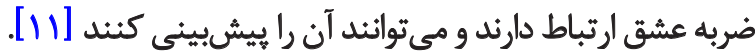

2. Engel

3. Personality factors

4. Five factor model

5. Bowlby

6. Myhr

7. Mikulincer \& Shaver 
مقياس شخصيت هيَّزاكو"

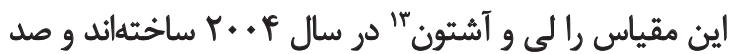

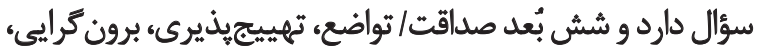

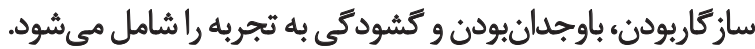

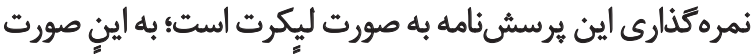

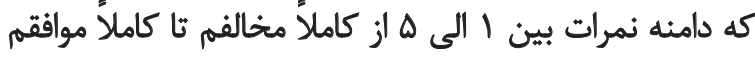

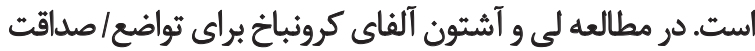

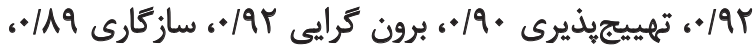

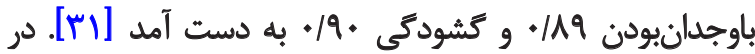

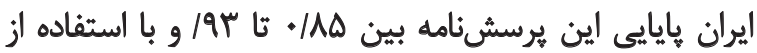

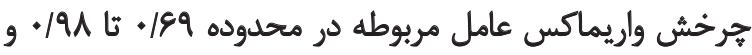

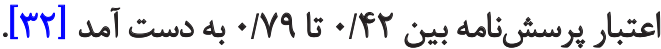

\section{نسخه كوثاه مثياس طرحواره يانتَّ1}

اين مقياس راكه بر بايه مشاهدات تجربهشدهاست متخصصان

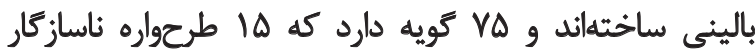

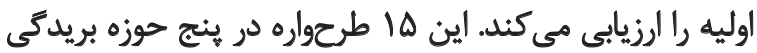

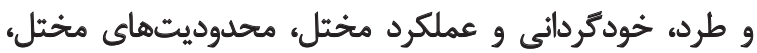

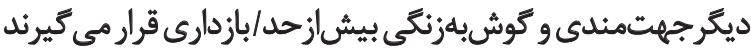

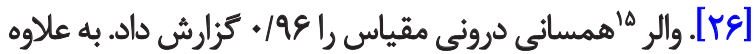

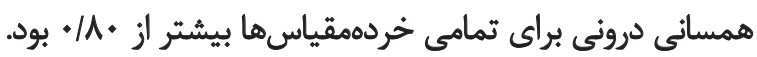

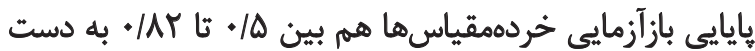

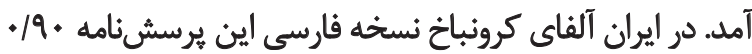

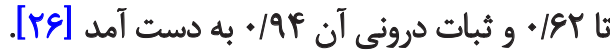

$$
\text { مقياس دلبستئى والدين و همسالاني" } 17
$$

اين مقياس را آرمسدن" در سار سال 191V به صورت

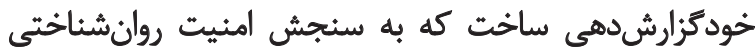

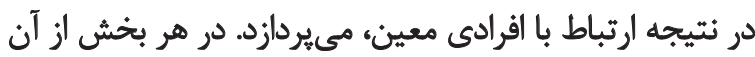

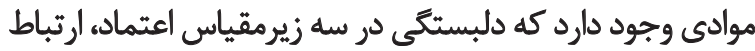

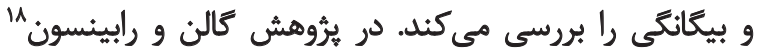

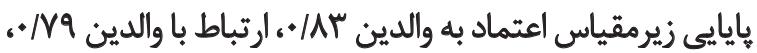

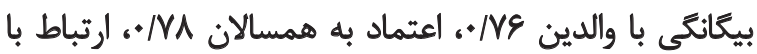

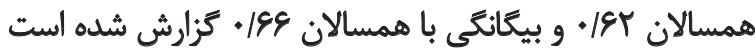

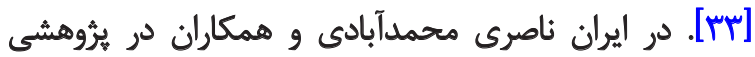

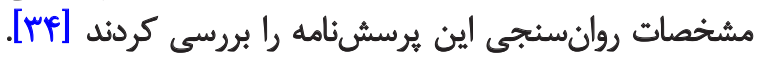

\section{HEXACO-PI-R}

13. Lee \& Ashton

14. Young Schema Questionnaire-Short Form (YSQ-SF)

15. Waller

16. (IPPA-R)

17. Armsden

18. Gullone \& Robinson

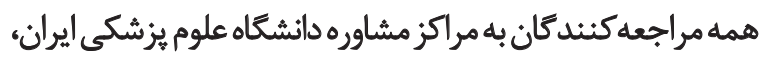

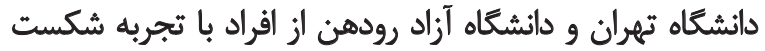

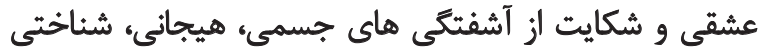

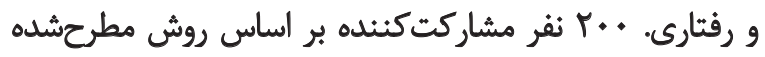

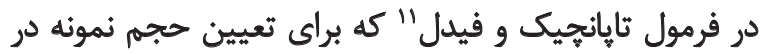

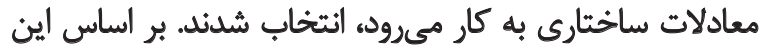

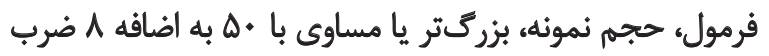

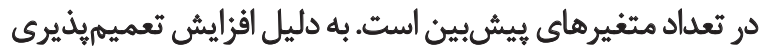

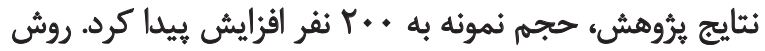

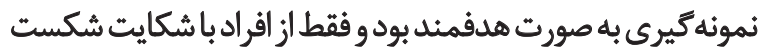

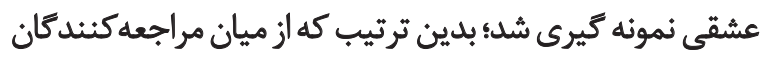

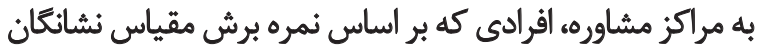

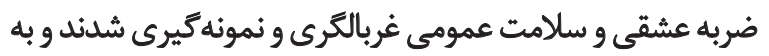

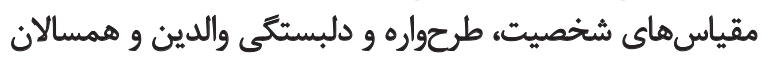

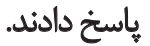

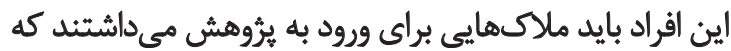

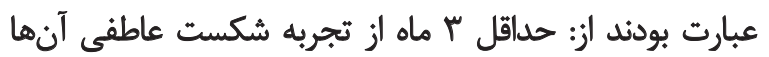

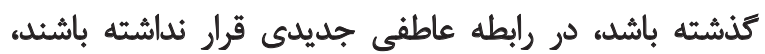

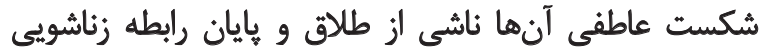

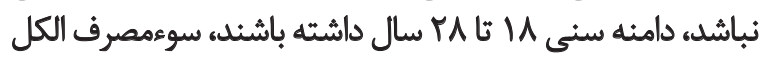

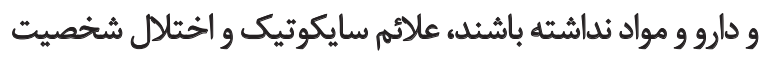

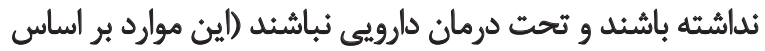
يرونده دانشجويى و به تشخيص روانيزشك سنان سنجيده شاند).

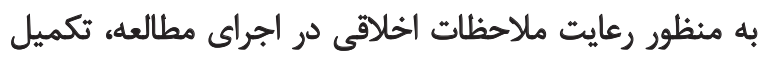

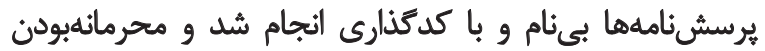

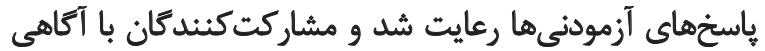

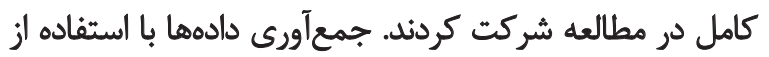

$$
\text { مقياس هاي زير انجام شد. }
$$

$$
\text { مقياس نشانكًان ضربه عشئق }
$$

اين مقياس را راس در سال 1999 براى سنجش شدت

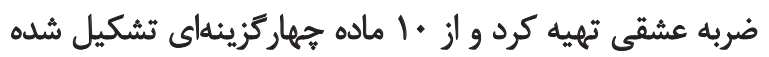

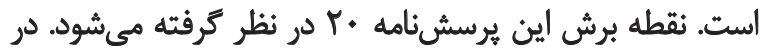

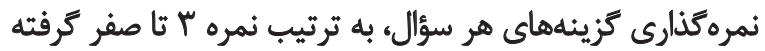

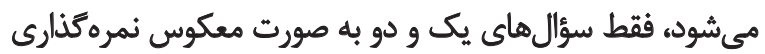

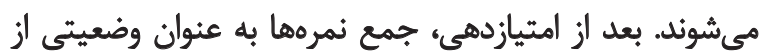

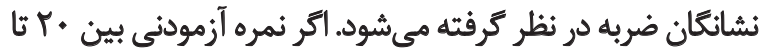

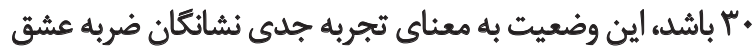

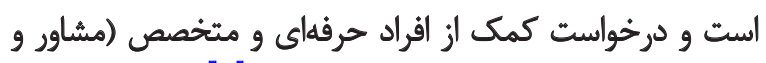

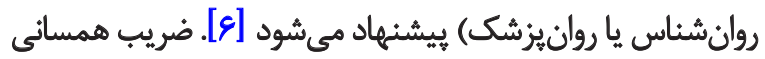

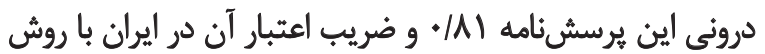

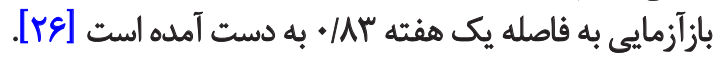


بود. در ادامه يافتههاى توصيفى و استنباطى بررسى مى شوندئ.

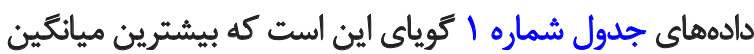

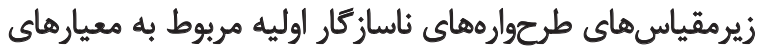

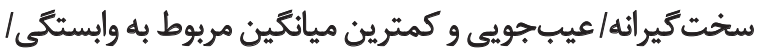

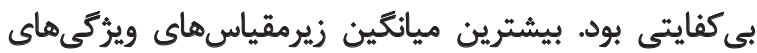

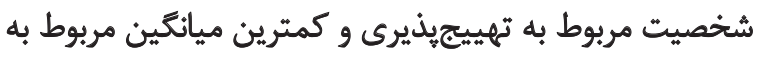

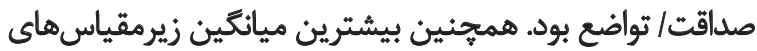
كيفيت روابط دلبستَّى مربوط به ارتباط بـا همسالان و كمترين

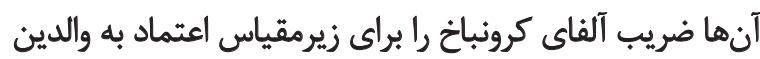

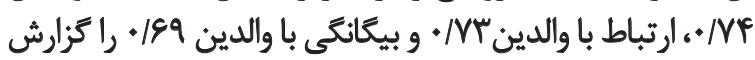

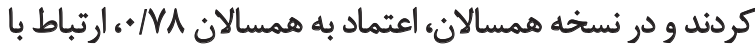

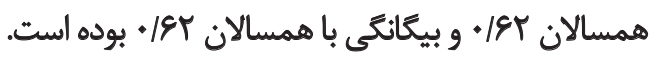

ياقتهها

در اين يُروهش • •r مشاركت كننده با اظهار شكست عاطفى شركت كرده بودند كه ميانكين سنى آنها

جدول ا. مشخصههاى آمارى (ميانكين و انحراف معيار) متغيرهاى يزؤهش

\begin{tabular}{|c|c|c|}
\hline ميانكين +انحراف استاندارد & زيرمقياسها & متغيرها \\
\hline $\mid r / 9 \Delta \pm \varepsilon / q 1$ & محروميت هيجانى & \multirow{15}{*}{ 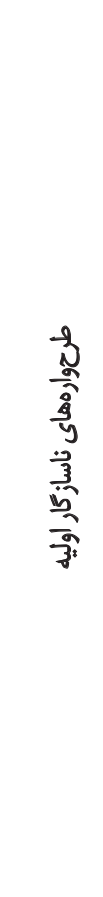 } \\
\hline $\mid r / q \Delta \pm \Delta / \Delta r$ & رهاشدكى/بىثباتى & \\
\hline$|r / \cdot| \pm E / P \mid$ & بيىاعتمادى/بدرفقتارى & \\
\hline $1 . / r \Delta \pm \Delta / r r$ & انزواى اجتماعى/بيكانكى & \\
\hline $\mid r / q \Delta \pm \Delta / \Delta \Lambda$ & نقص/شرم & \\
\hline $1 . / 1 r \pm \varepsilon / \pi$. & شكست & \\
\hline$q / n \pm \pm / s$. & وابستكى/بى كفايتى & \\
\hline$W / / \gamma \pm \Delta / V \varepsilon$ & آسيبيذيرى به ضرر يا بيمارى & \\
\hline $1 . / Q \subseteq \pm \& / \Delta \Delta$ & خود تحولنايافته/ كرفتتارى & \\
\hline $\mid r / A q \pm \Delta / \Delta \Lambda$ & |طاعت & \\
\hline $\mid V / q \pm \pm \Delta / \wedge q$ & ايثار & \\
\hline $\mid H / r q \pm g / \Delta F$ & بازدارى هيجانى & \\
\hline WOA \pm \&/qF & هعيارهاى سخت كير|ثه/عيبجويع & \\
\hline W/.EtY/Fa & استحقاق/بزركمنشى & \\
\hline $\mid r / q Y \pm \Delta / \Delta V$ & خويشتندارى/ خودانضباطى ناكافى & \\
\hline pe/vativ/1q & صداقت/تواضع & \multirow{6}{*}{$\begin{array}{l}x^{2} \\
y^{2} \\
y^{3} \\
y \\
y^{3} \\
3 \\
y\end{array}$} \\
\hline$\Delta \Delta /|\Psi \pm q / T|$ & تمييجيذيرى & \\
\hline$\Delta V / A V \pm 1+/ 9 V$ & برونكرايع & \\
\hline$\Delta T / M I T / F E$ & 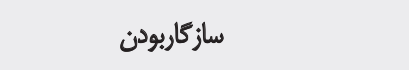 & \\
\hline$\Delta \Psi / T Y \pm V / \Delta Q T$ & باوجدانبودن & \\
\hline$\Delta r \pm V / a Y$ & كشودكى به تجربه & \\
\hline$W W \pm ז / q \Psi$ & اعتماد به والدين & \multirow{6}{*}{ 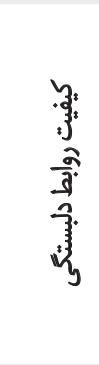 } \\
\hline WMETrar & ارتباط با والدين & \\
\hline$W / / Y \pm F / a r$ & بيكانكى از والدين & \\
\hline $1 V \cdot V \pm F / / r$ & ارتباط با همسالان & \\
\hline WAVEYr/M & اعتماد به همسالان & \\
\hline W/SET/RV & بيكانكى با همسالان & \\
\hline $\mid Q / A F \pm R / R T$ & شدت ثشانكان ضربه عشقىى & \\
\hline
\end{tabular}


جدول r. شاخصهاى نيكويى برازش مدل ساختارى

\begin{tabular}{|c|c|c|c|c|c|c|}
\hline IFI & NNFI & NFI & AGFI & GFI & RMSEA & شاخص برازندكى \\
\hline+-1 & $>+/ 9$ & $>. / 9$ & $>. / 9$ & $>. / 9$ & $<\cdot / 1$ & مقادير قابل قبول \\
\hline .191 & .191 &.$/ 98$ &.$/ 94$ &.$/ 9 \Delta$ & .1 .07 & مقادير محاسبهشهده \\
\hline
\end{tabular}

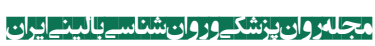

توزيع همه متغيرها عادى است؛ بنابراين مىتوان از آزمونهاى

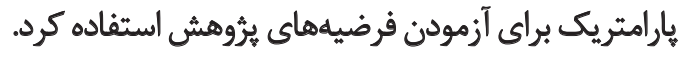

براى سنجش روابط بين ويزٔى هاى شخصيتى، طرحوارههاى

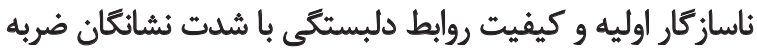

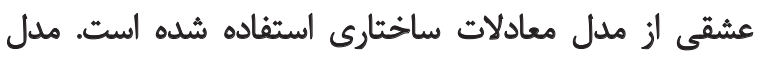

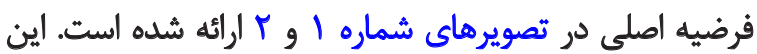
مدل با اقتباس از برون داد نرمافزار ليزرل ترسيم شده است. أز آنجا

$$
\text { ميانكين مربوط به بيكانكى با همسالان بود. }
$$

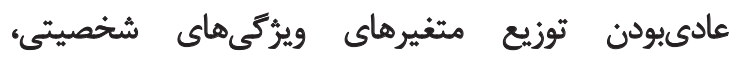

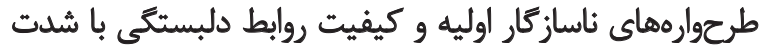

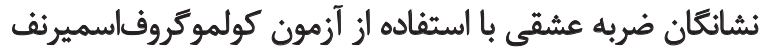

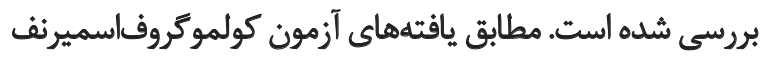

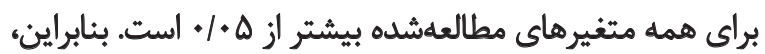
نتيجه آزمون براى هيجيك از متغيرها معنى دار نيست و درنتيجه

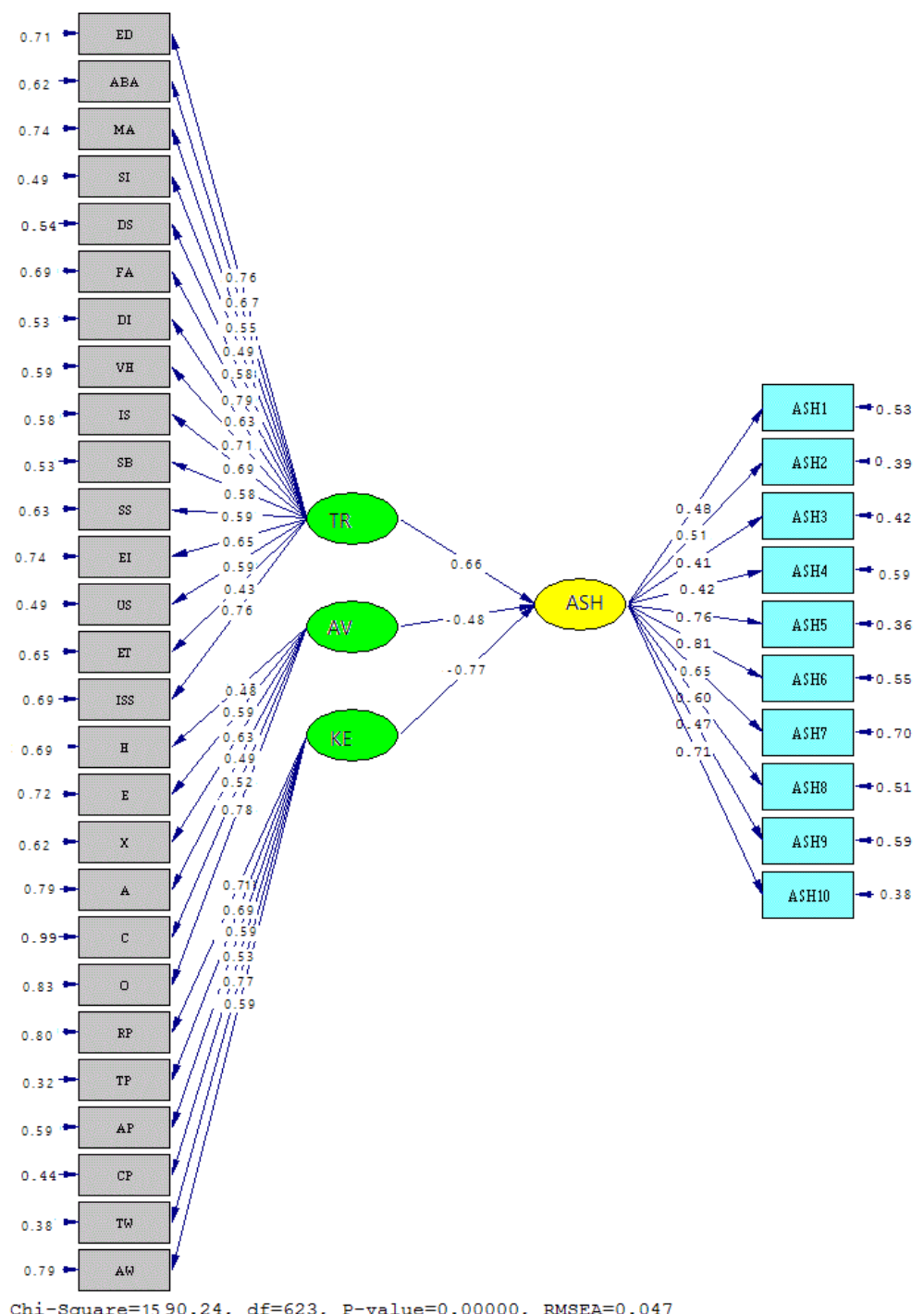

Chi-Square $=1590.24, \mathrm{df}=623, \mathrm{P}-\mathrm{value}=0.00000, \mathrm{RMSEA}=0.047$ 


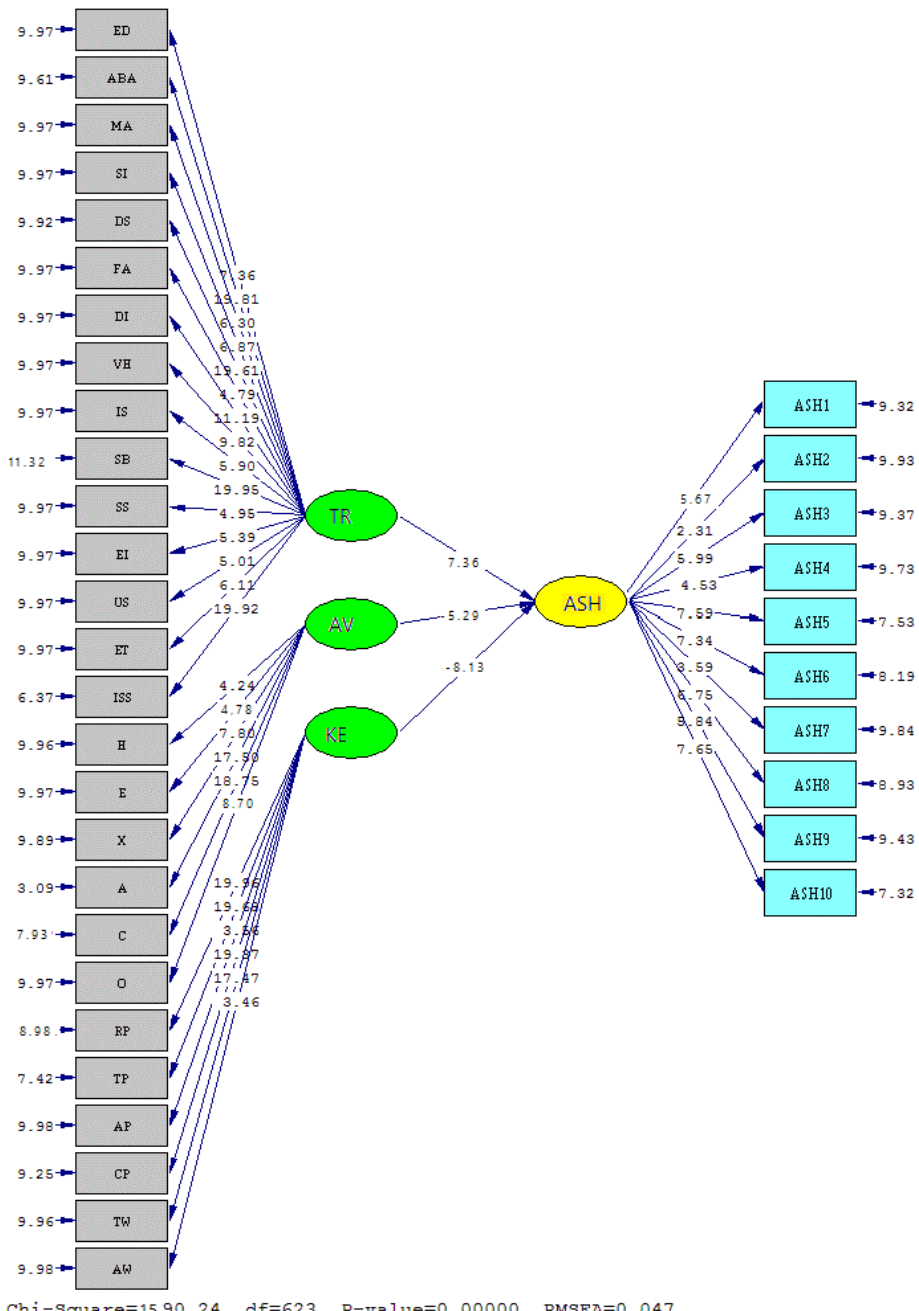

Chi-Square $=1590.24, \mathrm{df}=623, \mathrm{P}-\mathrm{value}=0.00000, \mathrm{RMSEA}=0.047$

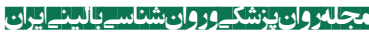

مى توان كفت ويرُكى هاى شخصيت با نشانكان ضربه عشقى رابطه

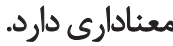

از طرفى بر اساس تصويرهاى شماره إ و ب قدرت رابطه ميان

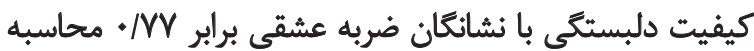

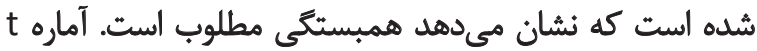

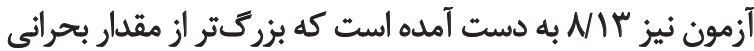

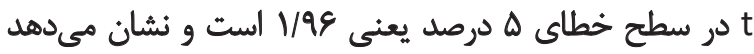

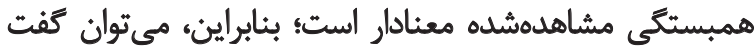

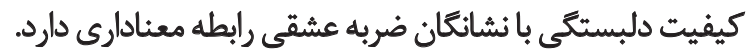

بحث

هدف اين يثروشش تدوين مدلى براى ييشيينى نشانكان

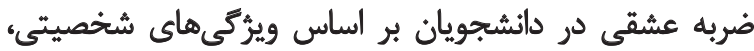

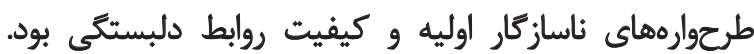

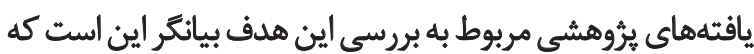

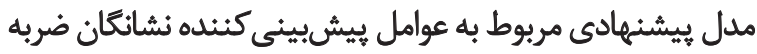

تصوير Y. آماره t نتايج تأييد مدل معادلات ساختارى

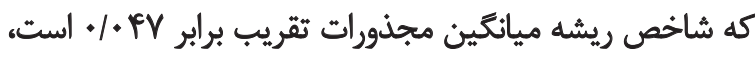

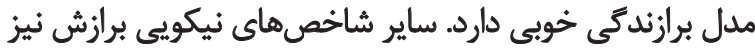

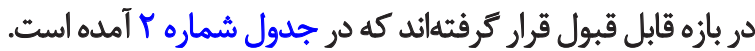
بر اساس تصويرهاى 1 و r قدرت رابطه ميان طححوارههاى

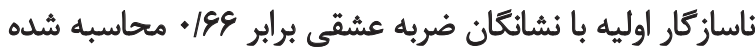

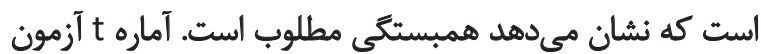

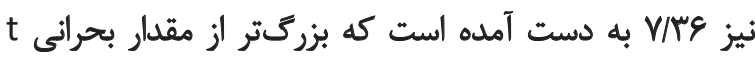

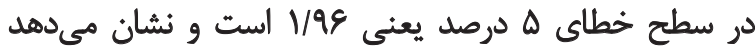

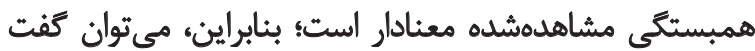

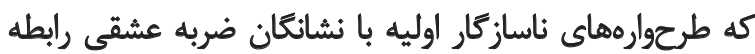

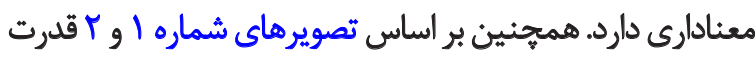

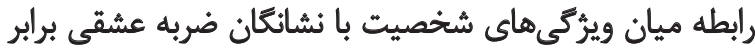

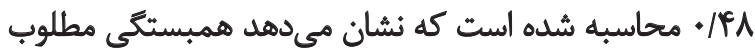

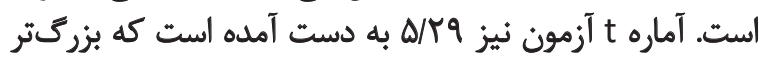

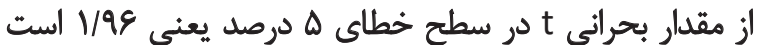

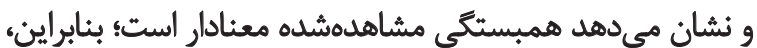




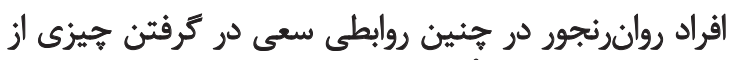

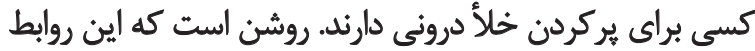

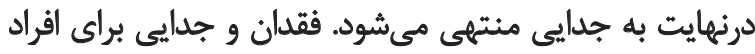

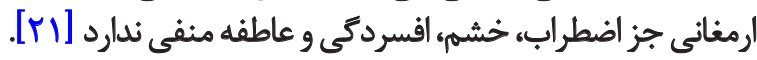
نتيجه اين يروهش نشان داد با كاهش متغير اعتماد به همسالان،

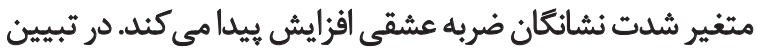

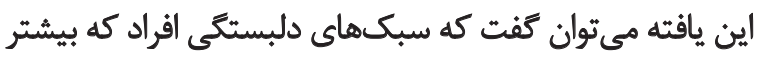
ناشى از سبكهاى ارتباطى استفادهشده از سوى مراقئ اقبان است،

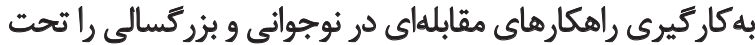

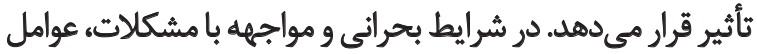

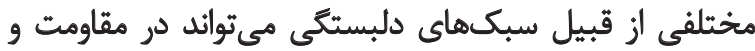

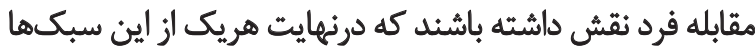

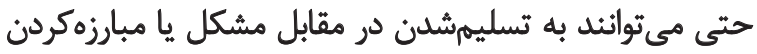

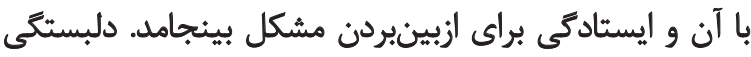

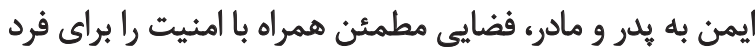

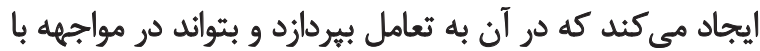

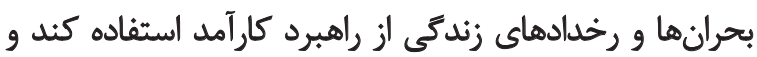

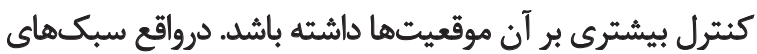

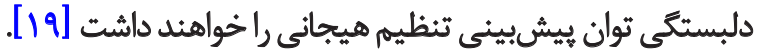

تنظيمهيجان انطباقى بهافراداجازهمي دهد تحمل وضعيتهاني

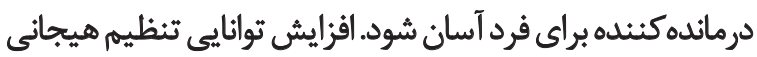

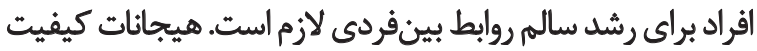

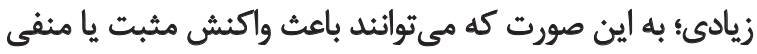

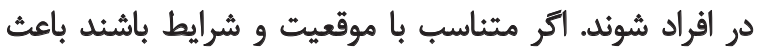

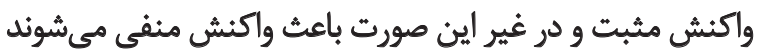

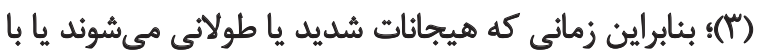

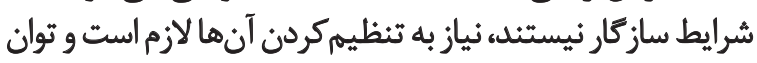

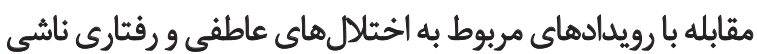

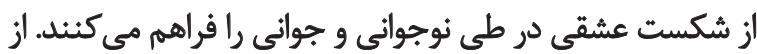

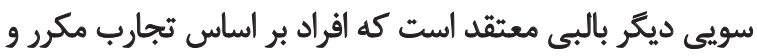

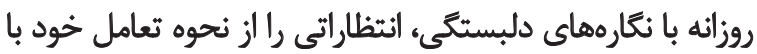

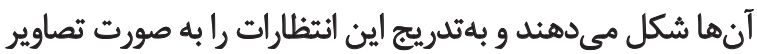

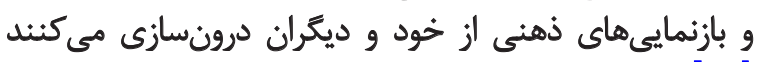

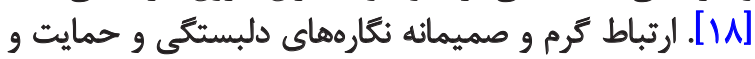

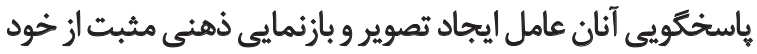

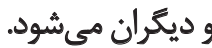

در اين صورت ديكران، از جمله والدين و همسالان به به صورت

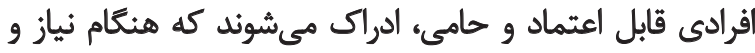

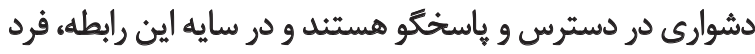

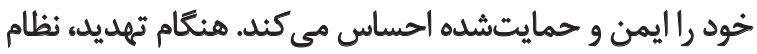

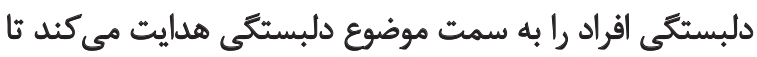

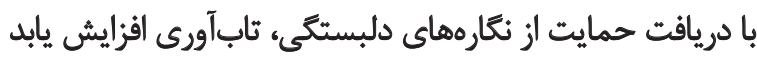

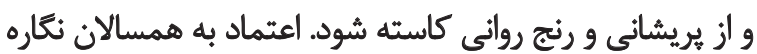

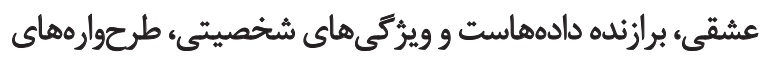

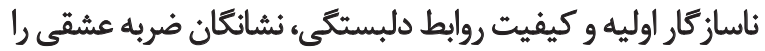

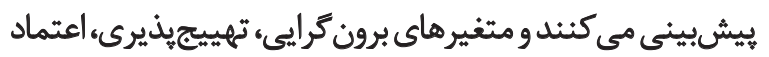

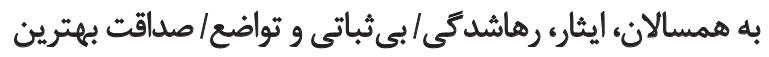
بيشبينى كنندهاى شدت نشانغان ضربه عشقى هستئد.

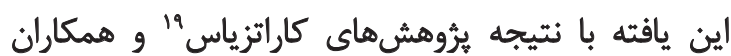

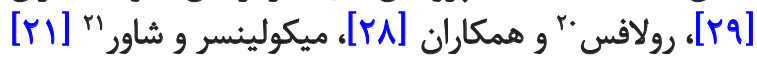

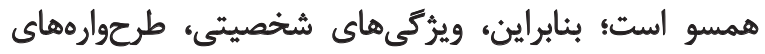

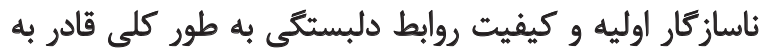

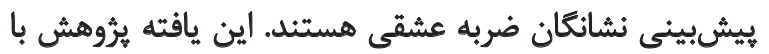

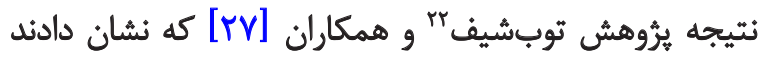

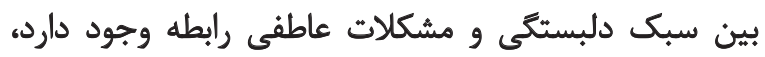

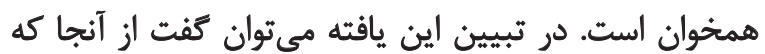

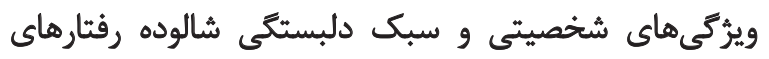

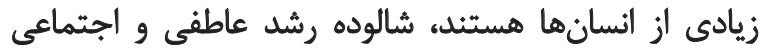
سالم در دوران بزركسالى را نيز يتىريزى مي كنئد.

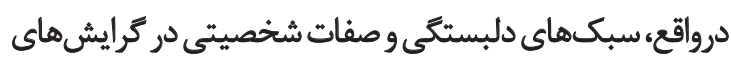

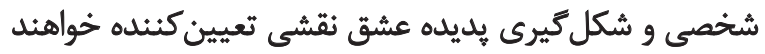

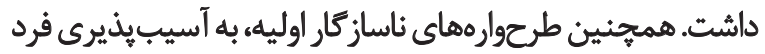

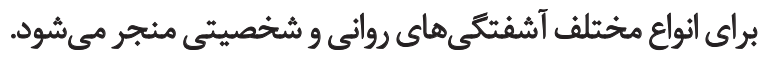

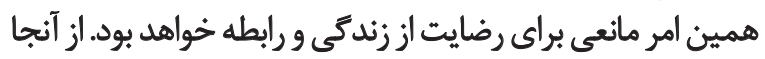

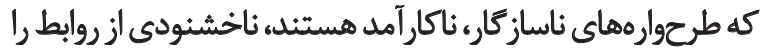

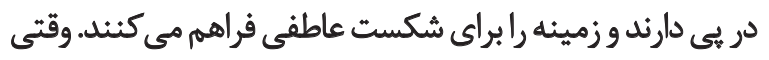

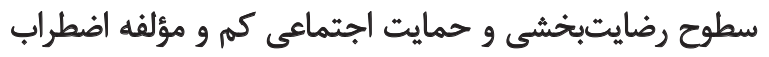

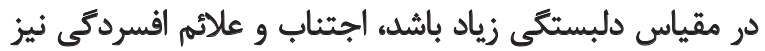

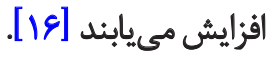

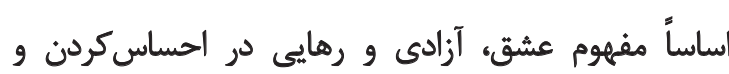

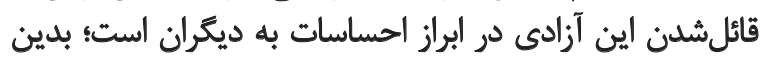

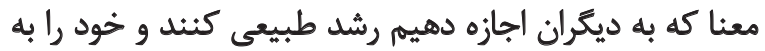

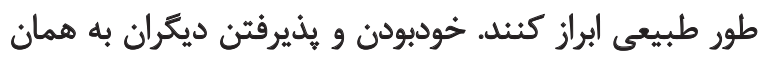
شكلى كه هستند لازمه عشق است. اشاره ضمنى ازئ إين اين تعريف

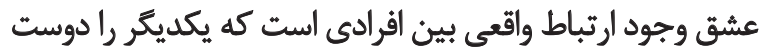

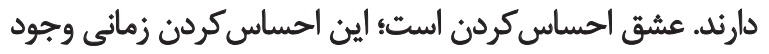

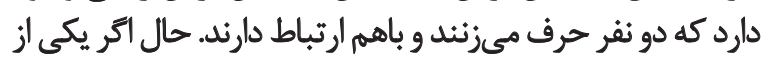

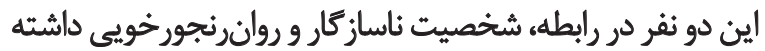

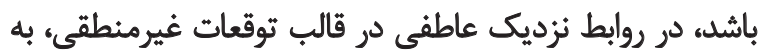

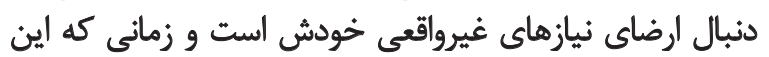

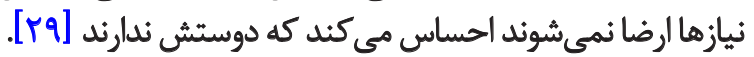

19. Karatzias

20. Roelofs

21. Mikulincer \& Shaver

22. Taube-Schiff 
رايج در ميان افراد جوان است، ولى اغلب اين افراد براي دريافت

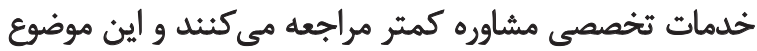

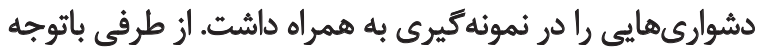

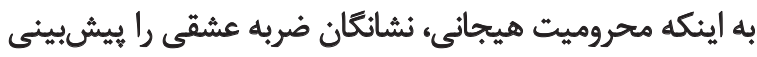

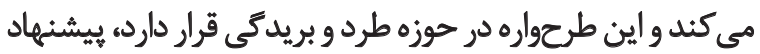

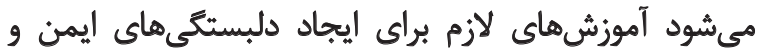

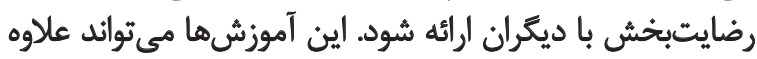

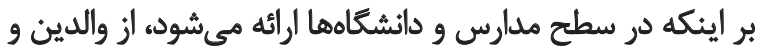
به واسطه شناخت شيوههاي معيوب فرزنديرورى و واصلاح الصاح آنها

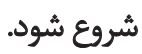

\section{مالاحظات اخلاقى}

\section{ييروى أز اصول اخلاق بثروهش}

همه اصول اخلاقي در اين مقاله رعايت شده است.

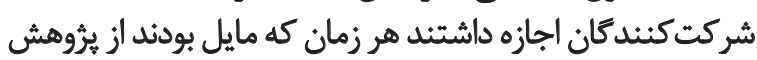

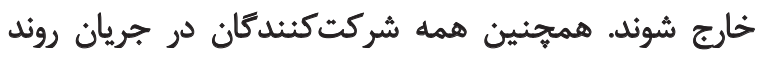

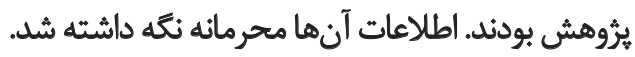

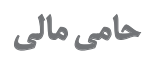

اين مقاله از رساله دكتراى نويسنده اول در گروه روانشناسي،

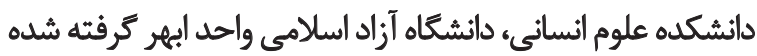

$$
\text { مشاركت نويسندكًان }
$$

مفهومسازى: تمام نويسندكان؛ روش شئاسي: تمام نويسندكان؛

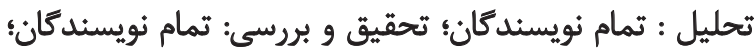

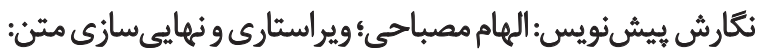

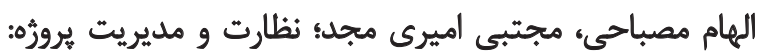

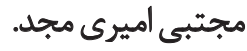

$$
\text { تعارض مئاقع }
$$

بنابر اظهار نويسندكان، اين مقاله تعارض منافع ندارد.
دلبستّى ايمنى را ايجاد هي كند باورهاى ارتباطى غيرمنطقىى

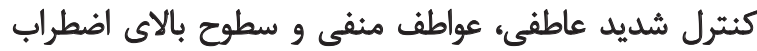

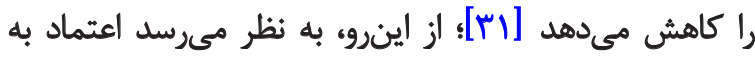
همسالان شدت نشانكان ضربه عشقى را كاهش إن دهد.

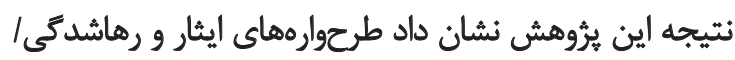

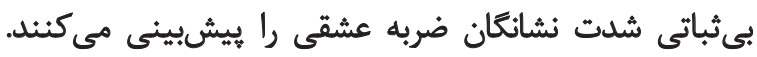

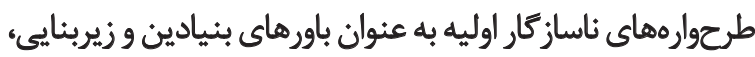

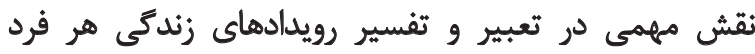

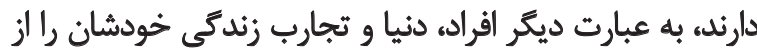

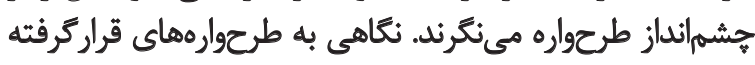

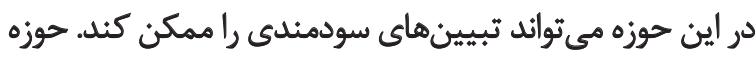

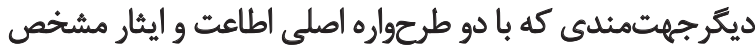

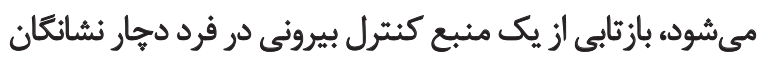

$$
\text { ضربه عشقى است. }
$$

ممكن است الين دو طرحواره با ناديدهانكارى نيازهاى هيجانى

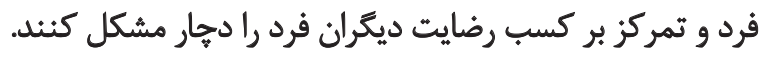

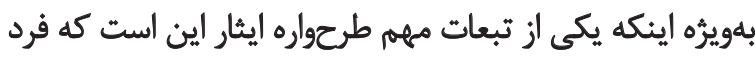

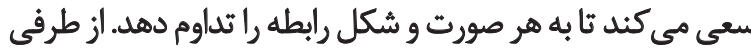

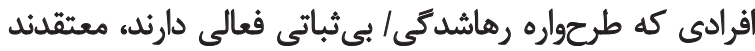

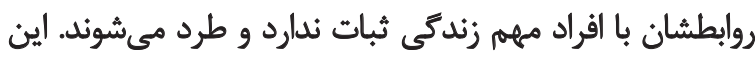

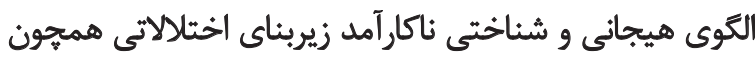

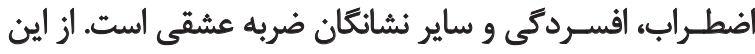

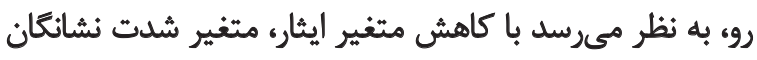

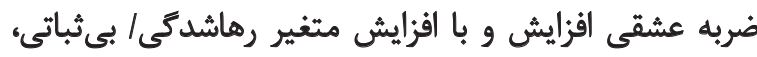
متغير شدت نشانكان ضربه عشقى افزايش ييدا كند.

\section{نتيجلكيرى}

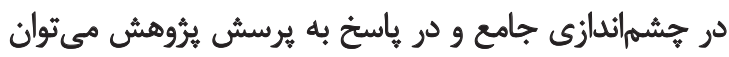

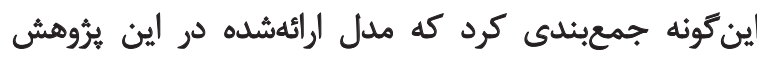

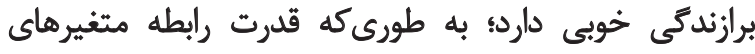

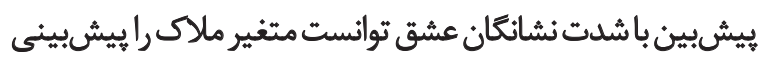

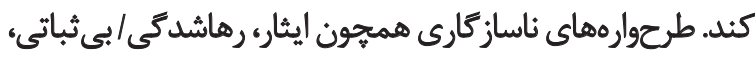

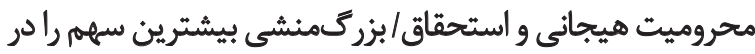

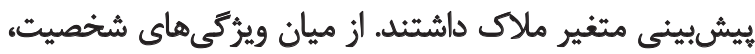

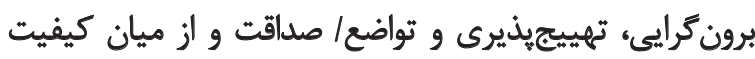

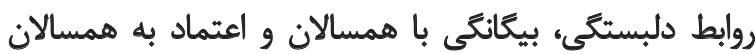

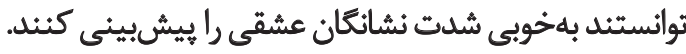

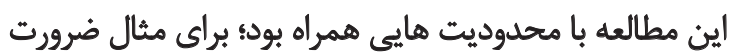

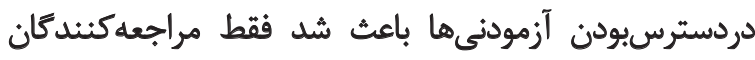

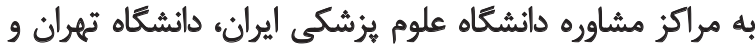

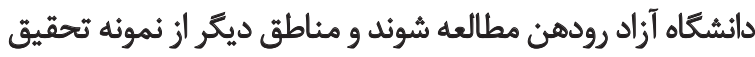

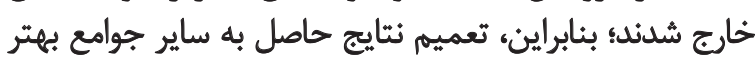
است با احتياط صورت گيرد. با اينكه شكست عشقي مسئ مسئلهاي 


\section{References}

[1] Moita G, Wieser MA. The dictatorship of love relationships. Zeitschrift für Psychodrama und Soziometrie. 2018; 17(1):21-32. [DOI:10.1007/s11620-017-0425-6]

[2] Prelipcean T. Saint Augustine-the apologist of love. ProcediaSocial and Behavioral Sciences. 2014; 149:765-71. [DOI:10.1016/j. sbspro.2014.08.309]

[3] Simon RW, Barrett AE. Nonmarital romantic relationships and mental health in early adulthood: Does the association differ for women and men. Journal of Health and Social Behavior. 2010; 51(2):168-82. [DOI:10.1177/0022146510372343] [PMID]

[4] Rhoades GK, Kamp Dush CM, Atkins DC, Stanley SM, Markman HJ. Breaking up is hard to do: The impact of unmarried relationship dissolution on mental health and life satisfaction. Journal of Family Psychology. 2011; 25(3):366-74. [DOI:10.1037/a0023627] [PMID] [PMCID]

[5] Sbarra DA, Emery RE. The emotional sequelae of nonmarital relationship dissolution: Analysis of change and intraindividual variability over time. Personal Relationships. 2005; 12(2):213-32. [DOI:10.1111/j.1350-4126.2005.00112.x]

[6] Rosse RB. The love trauma syndrome: free yourself from the pain of a broken heart. Boston: Da Capo Lifelong Books; 2007.

[7] Wilde JL. It's not me it's you: Examining the link between partner-schema organization, relationship functioning, and depressive symptoms [MSc. thesis]. Ontario: Western University; 2017.

[8] Young JE, Klosko JS, Weishaar ME. Schema therapy: A practitioner's guide. New York City: Guilford Press; 2003.

[9] Calvete E, Fernández-González L, González-Cabrera JM, Gámez-Guadix M. Continued bullying victimization in adolescents: maladaptive schemas as a mediational mechanism. Journal of Youth and Adolescence. 2018; 47(3):650-60. [DOI:10.1007/ s10964-017-0677-5] [PMID]

[10] Sempertegui GA, Karreman A, Arntz A, Bekker MH. Schema therapy for borderline personality disorder: A comprehensive review of its empirical foundations, effectiveness and implementation possibilities. Clinical Psychology Review. 2013; 33(3):426-47. [DOI:10.1016/j.cpr.2012.11.006] [PMID]

[11] Yousefi R, Adhamahian E, Afnhglmhamane K. [The role of early maladaptive schemas, personality traits and attachment styles in prediction symptoms of female students love trauma syndrome (Persian)]. Quarterly Journal of Women's Studies Sociological and Psychological. 2017; 14(3):171-90.

[12] Jovanović D, Lipovac K, Stanojević P, Stanojević D. The effects of personality traits on driving-related anger and aggressive behaviour in traffic among Serbian drivers. Transportation Research Part F: Traffic Psychology and Behaviour. 2011; 14(1):4353. [DOI:10.1016/j.trf.2010.09.005]

[13] Hosseini FS, Darvishi K. [The role of personality characteristics and parenting dimensions in Self-immolation and adolescent selfesteem in teenagers (Persian)]. Cultural-Educational Quarterly of Women and Family. 2015; 10(32):73-98.

[14] Selden M, Goodie AS. Review of the effects of five factor model personality traits on network structures and perceptions of structure. Social Networks. 2018; 52:81-99. [DOI:10.1016/j.soce net.2017.05.007]
[15] Bagby RM, Widiger TA. Five factor model personality disorder scales: An introduction to a special section on assessment of maladaptive variants of the five factor model. Psychological Assessment. 2018; 30(1):1-9. [DOI:10.1037/ pas0000523] [PMID]

[16] Kim S, Sharp C, Carbone C. The protective role of attachment security for adolescent borderline personality disorder features via enhanced positive emotion regulation strategies. Personality Disorders: Theory, Research, and Treatment. 2014; 5(2):125-36. [DOI:10.1037/per0000038] [PMID]

[17] Burke L. Growth Psychology (from fertilization to childhood) [YS Mohammadi, Persian trans.]. Tehran: Arasbaran; 2011.

[18] Kennedy M, Betts L, Dunn T, Sonuga-Barke E, Underwood J. Applying Pleck's model of paternal involvement to the study of preschool attachment quality: A proof of concept study. Early Child Development and Care. 2015; 185(4):601-13. [DOI:10.1080/ 03004430.2014.944907]

[19] Sepehrian Azar F, Asadi Majreh S, Asadnia S, Farnoodi L. [The relationship between attachment and coping styles with emotion dysregulation in adolescence (Persian)]. The Journal of Urmia University Medical Sciences. 2014; 25(10):922-30.

[20] Myhr G, Sookman D, Pinard G. Attachment security and parental bonding in adults with obsessive-compulsive disorder: a comparison with depressed out-patients and healthy controls. Acta Psychiatrica Scandinavica. 2004; 109(6):447-56. [DOI:10.1111/ j.1600-0047.2004.00271.x] [PMID]

[21] Mikulincer M, Shaver PR. Adult attachment orientations and relationship processes. Journal of Family Theory \& Review. 2012 4(4):259-74. [DOI:10.1111/j.1756-2589.2012.00142.x]

[22] Ho MY, Chen SX, Bond MH, Hui CM, Chan C, Friedman M. Linking adult attachment styles to relationship satisfaction in Hong Kong and the United States: The mediating role of personal and structural commitment. Journal of Happiness Studies. 2012; 13(3):565-78. [DOI:10.1007/s10902-011-9279-1]

[23] Lotfi N, Sa'atati Shamir A. [Investigating the relationship between personality traits and attachment styles with emotional failure (Persian)]. Clinical Psychology Research and Consulting. 2016; 6(1):112-98.

[24] Farrokhi Rad M, Arefi M. [Affectional qualities in girls with love affection syndrome (Persian)]. Paper presented at: National Conference on Future Studies, Humanities and Development. 11 November 2015; Shiraz, Iran.

[25] Tardust K. [The prediction of love blight syndrome based on self-sufficiency, attachment qualities and differentiation among female university students in Ahwaz (Persian)] [MSc. thesis]. Ahvaz: Shahid Chamran University of Ahvaz; 2014.

[26] Akbari E, Mahmoud Aliloo M, Khanjani Z, Poursharifi H, Fahimi S, Amiri Pichakolaei A, et al. [Early maladaptive schemas and parenting styles of students who have failed in love with and without the clinical syndrome (Persian)]. Iranian Journal of Psychiatry \& Clinical Psychology. 2012; 18(3):184-94.

[27] Taube-Schiff M, Van Exan J, Tanaka R, Wnuk S, Hawa R, Sockalingam $S$. Attachment style and emotional eating in bariatric surgery candidates: The mediating role of difficulties in emotion regulation. Eating Behaviors. 2015; 18:36-40. [DOI:10.1016/j.eatt beh.2015.03.011] [PMID]

[28] Roelofs J, Onckels L, Muris P. Attachment quality and psychopathological symptoms in clinically referred adolescents: The mediating role of early maladaptive schema. Journal of Child 
and Family Studies. 2013; 22(3):377-85. [DOI:10.1007/s10826-0129589-x] [PMID] [PMCID]

[29] Karatzias T, JowettS, Begley A, Deas S. Early maladaptive schemas in adult survivors of interpersonal trauma: Foundations for a cognitive theory of psychopathology. European Journal of Psychotraumatology. 2016; 7(1):30713. [DOI:10.3402/ejpt.v7.30713] [PMID] [PMCID]

[30] Fardis M. Expression and regulation of emotions in romantic relationships [PhD. dissertation]. Missoula, Montana: The University of Montana; 2007.

[31] Lee K, Ashton MC. Psychometric properties of the HEXACO personality inventory. Multivariate Behavioral Research. 2004; 39(2):329-58. [DOI:10.1207/s15327906mbr3902_8] [PMID]

[32] Fahimi S, Azimi Z, Akbari E, Amiri A, Pursharthi H. [Prognosis of clinical syndrome in students with experience of emotional breakdown based on personality structures: The role of moderating perceived social support (Persian)]. Journal of Knowledge and Wellness. 2014; 9(4):46-59.

[33] Gullone E, Robinson K. The Inventory of Parent and Peer Attachment-Revised (IPPA-R) for children: A psychometric investigation. Clinical Psychology \& Psychotherapy. 2005; 12(1):67-79. [DOI: 10.1002/cpp.433]

[34] Naseri Mohammad Abadi A, Askari A, Bahrami F, Sadegh $\mathrm{R}$, Abedi J. [Standardization of a questionnaire for parents 'and peers' attachment questionnaire in 9-11 year-old children in Isfahan (Persian)]. Clinical Psychology Research and Consulting. 2012; 2(2):25-40. 
This Page Intentionally Left Blank 